\title{
Los estudios sociales del comer: cultura, gusto y consumo
}

\author{
Aldana Boragnio \\ UBA (Universidad de Buenos Aires, Argentina) \\ IIGG (Instituto de Investigaciones Gino \\ Germani) \\ CIES (Centro de Investigaciones y Estudios \\ Sociológicos)
}

\section{Resumen}

Alimentarse es una conducta dirigida a obtener la energía para llevar a cabo las funciones que mantienen el funcionamiento del organismo a la vez que el desarrollo de sus capacidades físico-cognitivas. Sin embargo, el comer se constituye como un complejo sistema de relaciones socioculturales, de cohesión y de conflictividad. Por lo cual, para comprender por qué se come lo que se come debemos situar ese acto alimentario en un contexto, en una sociedad, un tiempo y un espacio determinado. En las ciudades el acceso depende del mercado y del Estado que compete a la producción, disponibilidad, circulación y consumo de alimentos.

El presente artículo busca plantear un recorrido teórico a partir de autores que nos permitan centrarnos en los cruces y entramados que se desprenden de la relación entre la comida como hecho cultural, el gusto como sentido organizado y configurado culturalmente

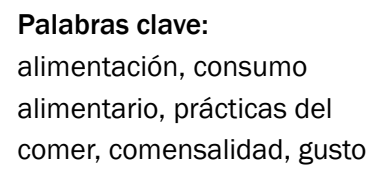


y el consumo alimentario en la conformación del comensal actual como objeto de investigación desde los estudios sociales.

\section{Abstract}

\section{Social studies of eating: culture, taste and consumption}

Feeding is a behaviour aimed at obtaining the energy to carry out the functions that maintain the organism running as well as the development of its physicalcognitive abilities. However, eating is constituted as a complex system of sociocultural relationships, cohesion and conflict. Therefore, to understand why you eat what you eat we must place this food act within a context, a society, a specific time and space. In cities, the access depends on the market and on the State which is the responsible for the food production, availability, circulation and consumption.

This article seeks to propose a theoretical journey from authors that allow us to focus on the intersections and frameworks that emerge from the relationship between food as a cultural fact, taste as a culturally organized and shaped sense, and food consumption in the creation of the current guest as a research object from social studies.

\section{Resumo}

\section{Os estudos sociais da alimentação: cultura, gosto e consumo}

Alimentar-se é um comportamento que visa obter a energia para desempenhar as funções que mantêm o funcionamento do organismo, como assim também como o desenvolvimento de suas habilidades físicocognitivas. No entanto, o comer constitui-se como um sistema complexo de relações socioculturais, de coesão e de conflito. Portanto, para entender por que se come o que come, devemos colocar o ato da alimentaçáo em
Keywords:

feeding, food consumption, eating practices, commensality, taste
Palavras-chave: alimentação, consumo de alimentos, práticas do comer, comensalidade, gosto 
um contexto, em uma sociedade, em um tempo e espaço específicos. Nas cidades, o acesso depende do mercado e do Estado responsável pela produção, disponibilidade, circulação e consumo de alimentos.

Este artigo procura propor um percorrido teórico de autores que nos permita focar nas interseçóes e estruturas que emergem da relação entre a comida como um fato cultural, o gosto como um sentido culturalmente organizado e configurado culturalmente, e o consumo de alimentos na conformação do comensal atual como objeto de pesquisa desde os estudos sociais.

\section{Introducción}

Desde el punto de vista arqueológico, histórico, antropológico, sociológico, biomédico, y hasta religioso, la alimentación fue y es una preocupación central para todas las sociedades. Mientras que el acto de alimentar(se) queda supeditado al plano bioquímico y fisiológico del organismo, desde el primer instante de vida el comer se constituye como un hecho cultural producto y productor de relaciones sociales. De este modo, la alimentación humana se conforma como un hecho complejo ya que se da en la tensión entre el cuerpo biológico individual que necesita ingerir nutrientes $y$ las relaciones sociales necesarias para que esto ocurra, a la vez que entre el placer individual y la necesidad biológica.

Alimentarse es una conducta dirigida a obtener la energía para llevar a cabo las funciones que mantienen el funcio- namiento del organismo a la vez que el desarrollo de sus capacidades físicocognitivas. Sin embargo, el comer se constituye como un complejo sistema de relaciones socioculturales, de cohesión y de conflictividad. Por lo cual, para comprender por qué, cómo, en dónde y con quiénes se come lo que se come debemos situar ese acto alimentario en un contexto, en una sociedad, un tiempo y un espacio determinado.

En las ciudades el acceso depende del mercado y del Estado que compete a la producción, disponibilidad, circulación y consumo de alimentos. Desde los últimos 20 ańos asistimos a un cambio en los discursos, las prácticas, los consumos, la producción y las políticas públicas en torno a la alimentación y la comida. La aparición de canales televisivos específicos sobre cocina, revistas, páginas webs, la proliferación de cuentas en redes sociales 
junto a los discursos de "alta cocina», vegetarianismo, comida "orgánica», junto al auge mediático de diversas dietas (veganismo, dieta "raw», lo "fit») y los movimientos en torno a estos discursos en todo el mundo, no solo tienen una impronta en el carácter nutricional, sino que evidencian sensibilidades, nuevas prácticas y lógicas de consumo que configuran relaciones sociales particulares y dinamizan diversos modos de distinción social.

Las urbes de la actual fase del capitalismo ofrecen múltiples versiones de vivencialidades diferenciales en las opciones de modo de vida, hábitos de consumo y normas culturales, confiriéndole a la calidad de vida urbana un aura de libertad de elección a la vez que se configuran como ciudades cada vez más divididas, fragmentadas y proclives al conflicto (Harvey, 20I3). Si hay una particularidad hoy en esta "ciudad colonial» (Quijano, 2000) en relación con el consumo de comida, es que conviven en el mismo espacio el FastFood, el SlowFood, las dietas vegetarianas, la comida orgánica, la comida ultra-procesada, la cocina de autor y los diversos tipos de hambre (De Castro, 1962), ${ }^{1}$ que posibilitan y configuran relaciones sociales particulares a partir de las distancias materiales y sociales que se consolidan.

En tanto la disponibilidad de recursos alimentarios altera las cantidades y calidades energéticas que cada individuo tiene a disposición, permitiéndole a esos cuerpos su reproducción y disponibilidad social, la alimentación será el punto nodal que permitirá comprender el sistema cultural de la sociedad, al mismo tiempo que las relaciones sociales que se desarrollan en ella (Scribano, 20I2). En la actualidad podemos ver la mercantilización tanto de los alimentos, produciendo disparidades mundiales referentes al abastecimiento y la accesibilidad de los mismos, como del comer como experiencias simbólicas; por lo que abordar el consumo de alimentos resulta un objeto significativo para los estudios sociales.

El presente artículo surge a partir del recorrido realizado en el proceso de investigación llevada adelante en 201720I8, titulada "Comer en la oficina: prácticas del comer y emociones de mujeres trabajadoras en el ámbito de la Administración Pública Nacional Argentina». En ella se abordaron tanto las estrategias de alimentación y del comer que las trabajadoras ponen en práctica en el ámbito laboral como las prácticas

1 El hambre es un fenómeno de gran variabilidad, el cual posee diversos matices. Entre los principales tipos de hambre que toma el régimen alimenticio actual se encuentran: el hambre aguda, el hambre crónica y el hambre oculta. Esta última está ligada a la malnutrición y refiere al hambre como un hecho primariamente social ya que es la forma de fabricación humana. 
de comensalidad y las emociones que se configuran a partir de allí. Esta investigación movilizó diversas actividades, lecturas y recursos que habilitaron el recorrido reflexivo presentado en estas páginas. Aquí, buscamos exponer una línea teórica en torno a los estudios sobre la alimentación, que remarque algunos conceptos necesarios para pensar la articulación entre comer, cultura y gusto, como una intersección de importancia para los estudios sociales y como una perspectiva a partir de la cual se pueden estudiar los cruces y entramados que surgen a partir de la confluencia necesaria de las ciencias sociales y humanas para la comprensión de las experiencias cotidianas de los sujetos.

La estrategia de presentación será la siguiente: a) presentaremos un recorrido teórico construido según un registro cronológico de los aportes los principales sobre la alimentación a partir de investigaciones socio-antropológicas, para luego proseguir con autores que configuran sub-campos temáticos sobre la alimentación; b) expondremos la relación entre la comida y la cultura, c) desarrollaremos brevemente algunos ejes sobre el gusto como un sentido geo-culturalmente localizado y d) desplegaremos las principales características del comer actual en la Argentina como una práctica ligada a la conformación de dos patrones de alimentación. Finalmente, esbozaremos unas palabras a modo de cierre.

\section{Alimentación y cultura: breve recorrido teórico}

El comer, la comida y las comensalidades son temas tratados desde diversos estudios socio-antropológicos que delimitaron y conceptualizaron las dimensiones de la alimentación como objeto de investigación. Entre ellos podemos remarcar los trabajos socioculturales de Norbert Elias ([1939]2009), quien estudió las modificaciones que sufrieron las prácticas cotidianas a partir del «proceso de pacificación", entendido este como el proceso de alejamiento progresivo del hombre de la naturaleza a partir de la gestión de las funciones corporales y de la regulación de sus emociones, el cual consta de procesos de autocontrol y autorregulación en la construcción del yo. En este proceso, las prácticas de alimentación son unas de las más relevantes, transformándose a partir de un conjunto de normas y reglas precisas sobre el «estar en la mesa».

Los trabajos de Lévi-Strauss (I968) son fundantes para el campo de estudio ya que expusieron al comer como un sistema cultural cristalizado en un triángulo estructural formado por lo crudo, lo cocido y lo podrido, el cual posee una dinámica particular en relación a las transformaciones culturales y naturales. Por su parte, Marvin Harris (1985), tomando a la alimentación como un sistema cultural, se centra en los procesos de diferenciación entre los alimentos los cuales se modifican en cada pueblo y 
cultura alimentaria. Así, el autor expone cómo los alimentos «buenos para comer» son los que presentan una relación costo/ beneficios más favorable para esa cultura. Desde estos procesos, el autor presenta a la alimentación como un proceso ecosocio-cultural.

Desde un enfoque sociológico, Jack Goody, en base a los trabajos de LéviStrauss, buscó comprender la relación entre la cocina y la clase social. A partir de un estudio comparativo, el autor mostró que en las sociedades con polarización entre los estratos aparecen las cocinas diferenciadas, pues se conforman una «alta» y una «baja» cocina. A la vez, hace hincapié en que esas cocinas no solo asumirán diferentes modos de combinación y de cocción de los alimentos, sino que también serán diferentes los alimentos y los productos utilizados, lo que dará lugar a distintos cuerpos de clase. En su libro Cocina, Cuisine y Clase: estudio de sociología comparada (1995), Goody pone especial énfasis en dos ejes: mostrar la relación entre la cocina y la sexualidad y la modificación que se dio en el formato de la alimentación a partir de la industrialización y de los procesos de conservación, de transporte y de venta minorista.

A partir de la ya establecida industrialización alimentaria, George Ritzer (1996), con el concepto de «McDonalización», se focalizó en el proceso de racionalización y en cómo estas prácticas se reprodujeron en toda la sociedad, tanto en los procesos laborales como en la alimentación. A partir del desarrollo de la industria de los alimentos se fue produciendo una racionalización no solo de la producción de estos, cada vez más rápidos de cocinar y de comer, sino también una globalización de la industria que promueve la homogeneización de los productos, de las industrias y del gusto, rompiendo con las diferenciaciones eco-socio-culturales.

Claude Fischler (1995, 20IO), por su parte, también se centró en el estudio de las normas de alimentación contemporáneas, pero marcando la desestructuración de ese "estar en la mesa», analizando las modificaciones de los lenguajes culinarios y la individualización de las normas que se dieron en las sociedades desarrolladas. Fischler, buscaba comprender al comensal actual y cómo esta modificación en las normas lo condicionaba; sin olvidar que identificó los procesos que se ponen en práctica para superarlas. Así, ya dando por sentada a la industria, se centra en el pensamiento clasificatorio del comensal. También, el autor expuso que, a través de la alimentación, ingerimos tanto nutrientes como sentidos y ello se debe al "principio de incorporación", el cual será central en la alimentación debido a que al comer incorporamos tanto lo material como lo simbólico del alimento.

Para comienzos del nuevo siglo diversos autores han indagado en el tratamiento de los cambios alimenticios de la llamada «modernidad alimentaria». La misma 
refiere al sistema de alimentación que se fue conformando en las sociedades industrializadas a partir de los cambios político-socio-económicos dados desde los años sesenta. Tiene como particularidades ser una alimentación globalizada, homogeneizada, industrializada, procesada, desestructurada e individualizada, la cual «se caracteriza por la proliferación de los miedos alimentarios y una arraigada sensación de inseguridad en relación a los alimentos que consumimos» (Contreras Hernández, 2005: 123).

Sabiendo de la existencia de una multiplicidad de investigaciones al respecto en diversas latitudes, ${ }^{2}$ y estando acotados en el espacio, en estas páginas nos centraremos en investigaciones de España y Argentina para acercarnos a las nuevas temáticas que se configuraron como objeto de investigación alimentaria a partir de la consolidación del campo y de los cambios que trajo el fin de siglo.

Desde España, Mabel Gracia Arnaiz (1997, 2003) se centra en las transformaciones tecnológicas, económicas, políticas y sociales que se dieron a partir de la década del sesenta y en cómo éstas condicionaron los comportamientos alimentarios. A la vez, desarrolla el papel de los medios de comunicación para divulgar, extender y profundizar estas variaciones. Luego, junto a Jesús Contreras Hernández
(2005), abordaron la investigación de la alimentación en un sentido amplio. Así, desarrollan diversos estudios tanto sobre los comportamientos socioculturales, los gustos y costumbres actuales, el acceso diferenciado a los alimentos y la seguridad alimentaria, como sobre la alimentación como un fenómeno bio-cultural donde el organismo biológico necesita de alimentos para funcionar y estos condicionarán la forma y las enfermedades del cuerpo. Todo esto sin olvidar la influencia de la globalización de la industria alimenticia y los efectos que produjeron en las culturas alimentarias, principalmente en la homogeneización del sistema de representación y en la confianza/desconfianza por la que transitan los comensales.

A su vez, Cecilia Díaz-Méndez y González-Álvarez (2013) observan cómo el discurso publicitario constituye el puente entre la industria alimenticia y los consumidores, otorgándole la legitimidad necesaria, a la vez que dirige las publicidades a diferentes grupos poblacionales y contribuye a la producción de nuevos hábitos de alimentación en los comensales.

Cáceres Nevot y Espeitx Bernat (2010), por su parte, trabajaron sobre cómo se piensa y vive la alimentación en el siglo XxI a través de la tríada comensal/ciudadano/consumidor en la que el comensal

2 Para ver más: Lupton, 1996; Aries, 1997; Ascher, 2005; Mestdag, 2005; Hernández y Villaseñor, 2014; Warde, 2017 
no es solo quien comparte la mesa, sino que es un sujeto que comparte socialmente representaciones y pensamientos que organizan el comer de un modo y no de otro. Los autores dirán que esos modos llevarán a que el comensal/ciudadano, en relación con sus condicionantes prácticos, tenga comportamientos alimentarios cada vez más individualizados y que consuma en relación a ellos, pero expresando solo una parte de sus intereses, pensamientos y representaciones. En suma, a partir de estas articulaciones entre el comensal/el ciudadano/el consumidor, es posible observar nuevos modos de consumos que no deben ser considerados como faltos de coherencias.

En Argentina se viene trabajando la alimentación desde perspectivas socioantropológicas. Patricia Aguirre, realizó investigaciones que nos permiten comprender la alimentación y las estrategias de consumo diferenciadas por ingreso y cómo, desde allí, se conforma parte de la identidad (2009, 2005, 1997). También, se dedicó a observar los cambios que se produjeron en el patrón alimentario y los impactos en la alimentación de las políticas económicas implementadas desde fines de los años ochenta (2004). Aguirre profundizó en la conformación de los cuerpos desde la alimentación y en las modificaciones que se generaron en éstos a partir de los cambios de la modernidad alimentaria, lo que tuvo como resultado el fenómeno actual de obesidad y las enfermedades crónicas no transmisibles -y la profundización de éstas en la pobreza- (2006).

Matías Bruera (2010, 2006), a través de estudios culturales, analizó el surgimiento del mundo gourmet en Argentina como contrapartida a la pobreza y al hambre. Desde la Sociología de los cuerpos y las emociones, Adrián Scribano profundizó en la vinculación entre la reproducción de los cuerpos, las emociones y la alimentación, al abordar la cuestión del hambre y el colonialismo como un problema social y geopolítico (20II, 20IO). Desde la misma perspectiva, Martín Eynard (20I7) investigó los conflictos sociales en torno a la alimentación durante el período de crisis y post-crisis económica en la ciudad de Córdoba. Eynard plantea que, cuando el conflicto alimentario fue tomado por la gestión del Estado, esto no implicó su solución sino que mutó a otras formas más atenuadas y menos visibles como son la obesidad en la pobreza, la malnutrición o «los petisos sociales». ${ }^{3}$

Es necesario destacar dos investigaciones claves en el desarrollo local. Primero, la investigación Remedi (2017, 2006) quien desarrolla el proceso de transformación económica, cultural y política que se dio

3 Este concepto se refiere a las personas de estatura baja debido a carencias alimentarias crónicas. 
en Argentina durante las últimas décadas del Siglo XIX, y cómo esto se asoció a la emergencia de una alimentación europeizada, construyendo dos dinámicas diferentes de consumo: el «afrancesamiento» de los consumos de los sectores acomodados $y$, por otro lado, un intercambio entre los sectores populares nativos y los colectivos migrantes de la época. ${ }^{4}$

Luego, la investigación llevada adelante por Patricia Aguirre, Diego Díaz Córdova y Gabriela Polischer (2015) en la que se detallan cuáles son los alimentos, los productos y los platos que se cocinan y se comen en Argentina actual. $\mathrm{Al}$ analizar las prácticas en el cocinar contemporáneo, el trabajo expone tanto el problema de la escasez alimentaria como el de la abundancia que, en contra de lo que se puede pensar, vuelve a las cocinas locales cada vez más monótonas y menos saludables. Al mismo tiempo, detallan los cambios en la consideración del evento alimentario al cual se le tiene una consideración aún menor, con pocas oportunidades de comer en grupo y en el hogar. Como resultado, los autores plantean la existencia de un consumo desregulado que lleva a la desintegración de los patrones alimentarios locales a la vez que articula con una cada vez mayor cantidad de patologías ligadas al consumo de alimentos.
Como podemos observar los análisis sobre alimentación van desde los comportamientos en la mesa como resultado de los procesos de estructuración social a éstos como modos de crear identidad. También encontramos una línea de trabajo ligada al consumo alimentario y las elecciones de los alimentos a partir del consumo. Aquí se hace hincapié en los aportes en relación a las crisis económicas y los conflictos sociales que se generan ante la caída de los ingresos de la población y los cambios en el patrón alimentario, pero, también podemos encontrar investigaciones que se centran en el gusto, las costumbres y las identidades para explicar la elección a nivel de los sujetos. Ahora, si observamos las investigaciones desde la presentación cronológica, podemos identificar no solo la instalación de la alimentación y sus prácticas como objeto de estudio sino también las nuevas lecturas que se necesitaron para dar cuenta dela incidencia de los procesos de individuación.

Lo que buscamos exponer con este recorrido es que la alimentación y el comer, se constituyen como un "campo dinámico» (Domaneschi, 2012), en el que se destacan tanto los amplios procesos de cambios históricos que les han sucedido como la complejidad de sus problemáticas actuales, pero siempre en relación a la 
cultura como eje organizador y de sentido de las prácticas.

A continuación, nos centraremos en la relación entre cultura y comida para luego, poner el ojo sobre el gusto como la explicación individual del comer, y sobre las dos grandes configuraciones del comensal a partir de la reorganización de los tiempos de ocio, trabajo y consumo.

\section{La comida como cultura}

A partir del descubrimiento del fuego (Goudsblom, 1995) y con la invención de la cocina, el comer se conformó en la actividad cultural por excelencia (Aguirre, 20I7; Jacinto García, 2013). Desde la antigüedad, la alimentación fue organizada de forma estrecha con la cultura y, principalmente, con la religión, generando una taxonomía de las especies que establecía la distinción entre puro e impuro. Luego, junto a la organización de lo permitido y lo prohibido a partir de la forma de obtención de los alimentos, de su combinación, preparación, distribución y conservación, da lugar a lo comestible y lo incomestible (Fischler, 20IO). Inicialmente, esta asignación se basaba en la disponibilidad de alimentos según zonas geográficas y climáticas, pero también en base a la carga simbólica que le imprime cada sociedad conformando luego, diferenciaciones jerárquicas que tienen sentido dentro de la organización de determinada cultura.

Actualmente, con el modelo de producción y consumo de alimentos a nivel global, los sentidos de la comida dejan de estar anclados a las limitaciones de su producción y consumo, pero se encuentra claramente diferenciados según el espacio social -y mundial- que se ocupe. Así, «luego de una primera división en la distribución, la cantidad, variedad y calidad de los alimentos, volverá a producirse un proceso de distribución diferencial» (Autor/a, 20I5: 184) a nivel global. En este sentido, comer es un proceso físicoorgánico, que está relacionado también con un tiempo, con una geografía, con una historia social y que, por lo tanto, va más allá de quien realiza tal acción.

Asimismo, no solo nos alimentamos, sino que elegimos qué comemos y ejercemos un tratamiento específico sobre lo que vamos a ingerir, es decir, lo seleccionamos, lo limpiamos, lo procesamos y lo cocinamos y, en este proceso de selección-procesamiento-preparación damos sentido a lo que ingerimos (Fischler, 1995). Esta conducta está organizada culturalmente y variará en cada sociedad, al tiempo que se verán modificadas de acuerdo al género, la edad, la posición social de quién coma, y el contexto específico de espacio/tiempo donde se llevará a cabo el acto de comer.

En los trabajos de Pierre Bourdieu (1998, 20Io) sobre la «distinción» y los "gustos», las prácticas culinarias, del comer y las estrategias alimenticias son distinguibles por los productos consumidos, que dependerán de los medios 
económicos y el capital cultural; por los modos de preparación, basados en la economía doméstica y la división de trabajo entre los sexos; a la vez que por las formas de comportarse en la mesa, que se distinguirán entre la ausencia o presencia de rituales específicos y de etiqueta. Observando así que a partir de la lógica de la distinción se pueden construir mapeos de las estructuras sociales.

Pero, estos habitus no solo son hábitos de gusto o alimentación, que pueden traer consecuencias nutricionales actuales y futuras, sino que estarán influenciando la proximidad de las relaciones sociales entre las personas, que se pueden expresar tanto mediante los tipos de alimentos y comidas que comparten juntos, como en la frecuencia de las mismas (Contreras, 1995).

Por consiguiente, se puede advertir la complejidad del evento alimentario, que se encuentra conformado bio-psicosocialmente y atravesado por variables culturales, económicas y políticas, de modo que se conforma como un "... símbolo de la civilización y la cultura...» (Montanari, 2004: 42), o como un "hecho social total» (sensu Mauss). En este sentido, el evento alimentario constituye un hecho complejo que se define como una experiencia central de la vida del ser humano, al mismo tiempo que es la primera relación que se establece con el físico que nos rodea.

\section{El gusto cultural del comer}

En el comer, de modo casi imperceptible y reforzándose en la cotidianeidad, se articulan las personas con la estructura social. Sin embargo, «en un acto de oscurecimiento digno de un mago» (Aguirre, 2017: 19), el comensal reducirá a su gusto, a su elección, a lo individual, lo que es condicionamiento social. Este acto «mágico» nos hace olvidar lo histórico-social de lo físico-biológico, que los sentidos son organizados, regulados y conformados socialmente en el proceso de las experiencias históricas que se in-corporan en la experiencia de la vida. Y estas formas constituirán tanto sociedad y cuerpo, como sensibilidades ${ }^{5}$ ya que «entre la sensación y la percepción, se halla la facultad de conocimiento que recuerda que el hombre no es un organismo biológico sino una criatura de sentido» (Le Breton, 2007: 22).

Dado que conocemos el mundo por y a través del cuerpo, el intercambio con el ambiente dará lugar a que los sentidos constituyan la base desde la que se hace posible la relación yo-mundo, la cual buscará adecuación de éstos a gestión de los cuerpos.

El gusto, es el sentido principal de esta relación ya que 
...exige la introducción en uno mismo de una parte del mundo. Los sonidos, los olores, las imágenes nacen fuera del cuerpo. Saborear un alimento o una bebida implica la inmersión de los mismos dentro de uno. La sensación aparece en la boca en el momento de la destrucción de su objeto, que entonces se mezcla con la carne dejando su huella sensible. (Le Breton, 2007:267)

A la vez, el gusto comparte con el olfato «el contacto con la cosa y su consecuente incorporación en la forma de una sensación dicotómica (bien/mal; fragante/ apestoso; rico/feo)» (Cervio, 20I5: 39), ${ }^{6}$ la cual dará la respuesta al por qué se come lo que se come, "porque es rico, porque gusta'. El gusto aparece así, como un sentido personal e incuestionable, ya que está «basado en criterios de elección individuales que permiten clasificar de manera inmediata e intuitiva los alimentos y comidas con los antitéticos y tautológicos: «me gusta», "no me gusta» (Lava, 20I2: 223). Pero quedar atrapado en la explicación de «me gusta»/«no me gusta" nos deja ubicados en un discurso individual que no nos permite captar lo relacional del sentido del gusto.

Así, en la medida en que entendemos a los sentidos como dependientes de las regulaciones sociales que se hacen cuerpo, estos se vuelven esenciales para indagar los procesos sociales. En este punto, es necesario afirmar que el gusto se conforma a partir de un proceso de aprendizaje social que se inicia desde la vida intrauterina, ${ }^{7}$ que continúa durante la infancia, se prolonga en el aprendizaje escolar, en las relaciones con pares durante la adolescencia y continúa activo durante toda la vida. Por ello en el sentido del gusto se puede observar los sistemas perceptivos tanto del hogar como de la experiencia social, ya que «no hay sentido más geoculturalmente determinado que el de la gustación, todos los localizadores de enclasamiento (clase, etnia, edad, género, etc.) atraviesan la vivencia del mundo a través del gusto" (Scribano, 2007: 6).

Pero, al hablar de gusto, se vuelve importante reforzar la idea de que "el órgano del gusto no es la lengua sino el cerebro, un órgano culturalmente (y por tanto históricamente) determinado» (Montanari, 2004: 55). Ahora, el cerebro no solo funciona como el órgano a partir del cual se aprenden, asimilan y transmiten los criterios de valoración, sino que el cerebro es el órgano a partir del cual se da la relación con el entorno, la conformación orgánica y filogenética del cuerpo. Específicamente,

6 Para ampliar la relación entre gusto y objeto ver, Autor/a, 2018.

7 En la vida fetal, la lengua y la nariz se encuentran bañados por el líquido amniótico que pasa por el área nasal, lo cual activa el sentido del gusto (Durán-Gutiérres et al, 2012: 140). 
a) Los cuerpos son tales, dada su conexión con el entorno/ambiente (condiciones materiales de existencia) a través de (y por) los complejos procesos que se generan en la interacción entre (con y desde) el cerebro/ sistema nervioso central/nutrientes/energías. b) En el cerebro se «alojan» (de modo complejo e indeterminado) los procesos de construcción social de los cuerpos y las emociones mediados por un conjunto de modularidades interactivas entre las «causas» químicas y eléctricas de los sistemas de vida que articulan las capacidades que poseen nutrientes/energías para posibilitar/ obturar, producir/reproducir y/o equilibrar/desequilibrar la existencia de esos cuerpos/emociones. c) La distribución y apropiación desigual de nutrientes/energías modelan las potencialidades que el sistema neurofisiológico tiene para «mantener» los estados de vida posibles de los sujetos en calidad de agentes sociales. d) Los procesos de estructuración social al «modelar» las conexiones posibles entre impresiones/ percepciones/sensaciones/emociones y cerebro/energías/ambiente son variables co-bordantes de las formas posibles de los cuerpos/emociones. (Scribano, 20I2: 97)

De este modo, es posible observar los límites de la sociedad que se hacen cuerpo, en tanto que los cuerpos serán producidos y reproducidos con los límites de las condiciones materiales de existencia que la sociedad distribuye desigualmente entre los sujetos. Los procesos que se dan en la interacción entre el cerebro, el sistema nervioso central, los nutrientes y las energías, dan lugar a la existencia y reproducción de los cuerpos. Esto no acerca a comprender la relación directa que se produce entre los nutrientes y las posibilidades de acción, ya que la energía social ${ }^{8}$ surge de esta relación. Por ello, la relación cuerpos/entorno/nutrientes es esencial para la conformación de los cuerpos, y ésta potenciará/obturará las capacidades tanto perceptivas como emocionales y cognitivas de esos cuerpos al crecer, con el gusto como justificador de las elecciones de alimentación cotidiana y de consumo alimentario al que accedan. Por lo tanto, la posibilidad y potencia para planear y ejecutar la acción estará condicionada por la disponibilidad de energías y, ésta, por la posibilidad de acceso a los alimentos.

Retomemos lo expuesto hasta aquí. Entendemos que el comer está atravesado por los sentidos y la gustación es uno de los primeros sentidos en ser regulado socialmente y, simultáneamente, el que

8 La energía social se basa en "la energía corporal y refiere a los procesos de distribución de la misma como sustrato de las condiciones de movimiento y acción. La potencia para planear, ejecutar y resolver las consecuencias de la acción de los agentes constituye la energía social que estos tienen" (Scribano y De Sena, 2016: 116). 
«justifica» la elección de la comida. En esta línea, es importante no perder de vista que el gusto se formará dentro de las pautas culturales que nos brinda la sociedad. Por consiguiente, a las reglas relativas de organización de las comidas, se le sumará un conjunto de normas que organizarán el gusto que explica el acto de comer. $\mathrm{Si}$ el gusto individual, refiere a una experiencia individual que se pierde, el gusto colectivo, es una «valoración sensorial» de lo bueno, malo, lo que gusta y lo que no, este es social y viene del aprendizaje, por lo tanto, es colectivo y comunicado (Montanari, 2004). Por consiguiente, el gusto es una experiencia cultural.

\section{Gusto, consumo y cultura}

En las sociedades industrializadas modernas, aparecieron nuevos comensales más ligados a la idea de "sujeto-consumidor» (Fischler, 1995). ${ }^{9}$ En este contexto donde el comensal deviene en consumidor, Gracia Arnaiz remarca que se tienden a conformar «nuevos grupos biosociales que comparten estilos de vida y gustos particulares atendiendo a las diferencias/ similitudes generacionales, de género o de seguimiento de modas más que a la adscripción de clase» (2003: 8). Pero, no hay que olvidar que estos nuevos modelos de consumo pueden estar sostenidos en «modelos de consumo de clase» a partir del cual también nos es posible podemos observar nuevas pautas de reproducción de la desigualdad por un acceso desigual a los nutrientes.

Así, la diferenciación del consumo entre clases se sustenta tanto en el volumen del capital (económico y cultural) como en la estructura del mismo. Por ello, es necesario pensar el consumo de alimentos como un conjunto de variables explicativas articuladas e interrelacionadas. Ello explica que, si la clase social nos permite comprender la heterogeneidad alimentaria en base al gasto alimentario y a la desigualdad en el acceso a ciertos alimentos, no puede explicar linealmente la pluralidad de pautas de consumo que se relacionan con los criterios de edad, salud y belleza. Aunque la relación "comensalidad-individuo-consumo» no es una relación lineal en la que el consumo alimentario se da solo en asociación a la posición social que reproduce las desigualdades, si se irán diferenciando dos patrones alimentarios, la comida de ricos y la comida de pobres (González Turmo, 1997; Aguirre, 2005).

Para acercarnos a resolver estas diferencias, entendemos que «existen fuerzas

9 El proceso de transformación del comensal a consumidor nos excede a los objetivos de estas páginas, pero es un tema interesante para pensar en consonancia con la conformación y crecimiento de la industria alimenticia y del marketing, los valores neoliberales y la extensión de la agricultura extensiva. Para ver más: Díaz-Méndez y González-Álvarez, 2013. 
sociales que van en una doble dirección (...): la individualización y la integración comunitaria, por un lado, y la estilización y la informalización, por el otro" (Warde, en Díaz Méndez, 2005: 59). La individualización hace referencia a la cada vez mayor cantidad de elecciones individuales que deben realizar los sujetos para sus menúes. La integración comunitaria se centra en los comportamientos alimentarios que generan vínculos que afirman la identidad. Del otro lado, la informalización, se entiende como la desestructuración de las comidas y la desregulación de las pautas que brindan apoyo para las elecciones; y la estilización refiere a nuevas reglas de consumo que se encuentran relacionadas con los gustos y que conforman «reglas estéticas propias de grupos sociales concretos» (Díaz Méndez, 2005: 60).

Otra de las características del comer actual es que esta nueva "libertad» de elección (la cual se encuentra guiada por la tendencia a la globalización y la homogeneidad de la oferta del mercado $)^{10}$ lleva incorporada una fuerte dosis de incertidumbre. $Y$ es que las personas deciden sobre su alimentación cotidianamente, pero cada vez cuentan con menos reglas y con más información —en este sentido, Fischler (1995: 195) desarrolló el concepto de "cacofonía», que se centra en las múltiples fuentes de información y en los discursos en relación a la comida (los culinarios, de nutrición y los gastronómicos) que ejercen el papel principal en el conocimiento de lo referido a la alimentación.

Hablando sobre las diferencias estructurales en la alimentación, también es necesario remarcar que la identidad social se define y afirma en las diferencias en donde los que piensan y comen como nosotros se distinguen y separan de «los que no son, no comen, no piensan como nosotros, es decir 'son los otros'" (Aguirre, 2009: 18); generándose un" «nosotros» cargado de connotaciones positivas. Este «nosotros», que posee un sistema de signos distintivos, variará según los estilos de vida y clasificará las prácticas y las percepciones de estas. Así quedarán también diferenciados los grupos sociales que poseen y defienden los mismos valores.

\section{Entre el comer gourmet y el comer lo que se pueda}

En Argentina, mientras en las clases medias y altas se individualizó el comer, a partir de los procesos de individualización, integración comunitaria, estilización e informalización de la modernidad

10 La industria homogeniza la oferta a nivel global al producir productos industriales que son iguales en todos los rincones del planeta, más allá de la geografía o de la cultura local. 
alimentaria, los rituales de la comida, se fueron relegando hacia al tiempo de ocio, convirtiéndose en un nuevo consumo cultural (Aguirre et al, 2015: 63). Así, la alimentación de estos estratos quedó estructurada en dos extremos: el de la carga y el de ocio. La comida cotidiana, que queda anclada en el trabajo, en la casa, en la simplificación de la estructura de la comida, en lo rápido y en el comer "cualquier cosita»; mientras que la extracotidiana se conforma como la «comida de disfrute» en lo que el consumo oficiará como organizador social.

La experiencia del comer, enmarcada en un tiempo y un espacio específico, se organiza en tanto sensibilidad gastronómica que funciona como diferenciador social. El hecho de "salir a comer", entendido como experiencia de clase, es «una práctica relacional ritual(izada) y mercantil(izada) que cobra sentido al estar-con-otros» (Lava, 2012: 215) y se conforma en la alta cocina, expandiéndose en la sensibilidad de la experiencia de "salir a comer», de las clases medias. Conformándose así un nosotros que, si bien quizás no entiende del «buen vivir», sabe de las experiencias legítimamente valoradas. En este caso, la práctica del comer transciende la necesidad de ali- mentarse, cargándose de normas, pautas, estilos y estéticas que transforma a esa práctica, y el concepto de «distinción» propuesto por Bourdieu nos permite pensar en el "salir a comer afuera», como una práctica que busca la relación de proximidad con la cultura legítima, en este caso con la sensibilidad del disfrute en el tiempo de ocio.

Esta conformación de dos tiempos sociales del comer es necesario ponerla en articulación con la historia reciente de la Argentina. Desde la década de los años 70, la pobreza no ha dejado de manifestarse como un problema de gran magnitud. Los niveles de la pobreza se dispararon desde principios de los años ochenta y se ubicó entre el 20 y el $35 \%$ de la población según el periodo de medición. Si bien, su crecimiento y fluctuación se vio ligada a los periodos de crisis económicas, los números nunca volvieron a los pisos estadísticos anteriores (De Sena, 2020). La variedad de respuestas dadas a esta problemática estructural estuvo de la mano del desarrollo de las políticas sociales alimentarias como modo de regular, normativizar y atenuar el conflicto (Sordini, 2018, Grassi, 2003).

Desde la década del ochenta, con la caja PAN ${ }^{11}$ como observable, se asiste a un

11 A mediados de 1984, durante el gobierno de Raúl Alfonsín, se puso en marcha el Programa Alimentario Nacional (PAN), el cual consistía en la entrega de bolsones de alimentos a familias que presentaban algún riesgo nutricional. Éste fue concebido como un programa de emergencia con dos años de duración, pero se mantuvo hasta el fin de su mandato. 
proceso de cambio en el patrón alimentario (que puede encontrar parte de su origen a nivel mundial con la Revolución verde), ${ }^{12}$ que se profundiza en la década del noventa y se instaló definitivamente luego de la crisis político-económica del 200I, transformando los modos de vinculación y las ideas en torno a la comida y las formas de comer. A partir del comienzo del siglo XXI, se produjo una crisis manifestada por la caída de la capacidad de acceso a los alimentos (el aumento de los precios, el desempleo y la disminución de los ingresos). El aumento en el costo de los alimentos produjo grandes transformaciones, los sectores medios y altos mantuvieron el patrón alimentario diversificando los consumos al compás del crecimiento de la oferta comercial, mientras los sectores menos favorecidos perdieron variedad en sus canastas de consumo (Aguirre, 2005: 98) "hasta que sus canastas resultaron suficientes en volumen, pero pobres en calidad de nutrientes» (Autor/a y Sordini, 2019: 72). Este proceso se puede observar en consonancia con la proliferación de restaurantes y de bares nocturnos con- centrados en el barrio de Palermo, que hacen la contraparte del periodo sociopolítico-económico del 200I-2003, ${ }^{13}$ en el que «mientras el hambre toma cuerpo de imagen costumbrista del paisaje, los paladares afinan sus gustos haciendo de la distinción de los sabores un valor agregado para el vínculo social y cultural» (Bruera, 2006: 26). Así, a la vez que los sectores sociales con mejores ingresos modificaron y diversificaron su alimentación, hacia el consumo de proteínas de origen animal (OMS/FAO, 2003; Aguirre, 20I5), los sectores de menores ingresos moldearon sus dietas en torno a las posibilidades de acceso, y esto se dio en conjunción con el mercado produciendo alimentos de baja calidad nutricional pero baratos para la distribución masiva y en relación al desarrollo de las políticas sociales alimentarias.

En este sentido, las prácticas del comer de los sectores más desfavorecidos de la estructura social se vieron modificadas por el quiebre del patrón alimentario y la conformación de dos sistemas de alimentación, a la vez que fueron modificándose por la nueva organización del

12 Revolución verde es la denominación usada internacionalmente para describir el importante incremento de la productividad agrícola de alimentos que se dio entre 1940 y 1970 en Estados Unidos, extendiéndose a varios países, y que consistió en la siembra de variedades mejoradas de maíz, trigo y otros granos bajo la forma de monocultivo, con la utilización de grandes cantidades de agua, fertilizantes y plaguicidas.

13 Para profundizar el análisis de la alta cocina y del "gusto" del estilo de vida de la comensalidad de Palermo, ver Lava y Saenz Valenzuela, 2012. 
tiempo que ya no se reguló a partir de las mismas lógicas de trabajo/ocio, sino que se configuran a partir de los tiempos de la relación escuela/comedor/asistencia.

De este modo, las distancias materiales y sociales de los diferentes sectores sociales se profundizan a partir de la distinción de la nueva sensibilidad gastronómica, ya que la pequeña burguesía encuentra el lugar perfecto para desempeñar el papel de vanguardia en todo lo que tiene que ver con lo que afecta al arte de vivir y centra su foco en la vida doméstica y en el consumo (Bourdieu, 1998: 45).

A partir de esos procesos, surge el mundo gourmet como un espacio fundamental en la estructuración de la mentalidad hegemónica contemporánea y urbana (Huergo y Bertone, 20I4: I47). Y, aunque podría parecer paradójico, no existe contradicción al respecto, ya que «lo gourmet» o la «Alta cocina» se instaló "como oclusivo respecto de la cuestión del hambre. El mundo gourmet se ha convertido en un programa, en una estética y en una ética frente a la desprotección, al hambre y al reparto de alimentos» (Bruera, 2007 en Huergo y Bertone, 20I4: 145). Dicho en otras palabras, el surgimiento de «lo gourmet» en el país se dio en el proceso en el que, mientras una parte de la población resultaba excluida de la comida, otro sector social refinaba su paladar seducido por la espectacularidad y la mercantilización tanto de los alimentos como del comer entendido como una experiencia simbólica sensible. Así, por un lado, encontramos el mundo gourmet y, por el otro, el mundo de los asistidos alimentarios, el cual, con el pasar de las crisis económicas y con la intervención del Estado a partir de políticas alimentarias asistenciales, ${ }^{14}$ vio modificadas sus prácticas individuales y colectivas de compartir y de gustar la comida y las formas de comensalidad (Cabral, Huergo e Ibañez, 20I2: I5), conformando un gusto de la necesidad (Bourdieu, 20I2: 44I).

\section{A modo de cierre}

Luego de lo expuesto, entendemos que comer es un acto que se relaciona con lo fisiológico-orgánico, pero atravesado necesariamente por su relación con la sociedad. Así, comemos comida, que son alimentos y nutrientes socializados a través de pautas culturales que los organizan en categorías y que los hacen comestibles.

Al mismo tiempo, si tenemos en cuenta que comer es una práctica esencial para la reproducción física, social y simbólica tanto del individuo como de la sociedad, la organización geopolítica de los alimentos condicionará la disponibilidad y el acceso a los mismos, por lo que hoy 
encontramos diversas formas del hambre en relación a la localización geográfica y social de los sujetos.

En la actualidad, por las características de las sociedades industrializadas en relación con el valor del tiempo, la tecnificación de la vida cotidiana y la cosificación del cuerpo, se vienen transformando continua y profundamente las formas del comer y del compartir la comida. Podemos observar una tendencia que se mueve hacia la individualización y hacia la desregulación de las pautas que estructuran el evento alimentario. Entre los principales cambios se observa el corrimiento de la elección de la comida al ámbito individual, la pérdida de los tiempos reglados de ingesta, el aumento de las veces y la cantidad de ingesta, la monotonía alimentaria y la pérdida del saber-hacer culinario. Así, la modernidad ha perdido los criterios por los que se explicaba y se transmitían las normas, pautas y reglas del comer, de este modo, la industria y el marketing reproducen las normas del comer a partir de la creación de un consumidor que busca parámetros cada vez más individuales y del disfrute que den sentido a su práctica.

Por otro lado, a la vez, se conforman comensales-destinatarios que comen lo que pueden, cuando pueden, en base a lo que la industria crea para ellos y a lo que los Estados le dan acceso.

Por todo ello, si aceptamos que el comer es un hecho socio-cultural que define la concordancia entre lo comestible y las reglas sociales que definen qué es un alimento y qué no, a la vez que organizan y rigen el cómo, cuándo, dónde, con quien, entendemos que el comer se encuentra hoy en una importante tensión. El comensal, devenido en consumidor, compra mercancías alimentarias que escapan a su saber y control. A la vez que, por otro lado, el comensal que no dispone del dinero para comprarlas, come lo que el Estado le entrega y a lo que le da acceso, a la vez que lo que el mercado produjo especialmente sin calidad nutricional. Esto explica que el comensal moderno de casi todos los estratos sociales, no sólo no puede controlar las variables que hacen comestible su comida, sino que las pautas culturales que conforman las reglas de la comensalidad ya no regulan el evento alimentario (Aguirre, 20I0: 35).

En resumen, entendemos que el comer, como actividad primordial para la vida individual y social, implica reglas y normas culturales que van más allá de la organización de qué es comida y qué no lo es. Por lo tanto, todo el acto de comer se encuentra organizado culturalmente, pero estas reglas se aprenden desde la primera socialización en el hogar, por lo que, se presentan como individuales y propias, de modo que se llega al punto en el que se oculta lo relacional del comer a partir de un discurso individual que explica que se come lo que se come, cuando se come y del modo en que se come porque "gusta». 
A partir de aquí podemos acercarnos a comprender la conformación de la multiplicidad de comensales-consumidores que se pueden encontrar en la actualidad, así como a sus características compartidas a la vez que, a sus diferencias, a sus estilos de vida y a la relación con el consumo, las clases sociales y las pautas de comensalidad que se modifican a partir de la modernidad alimentaria.

Esta relación entre comer, cultura, gusto y consumo, es una relación compleja donde se encuentran los aspectos materiales y simbólicos de la cultura, pero también las múltiples aristas de análisis para pensar el comer como un objeto sociológico central que posibilita y/u obtura la producción y reproducción del cuerpo tanto individual como social. Por ello, es necesario que los estudios sociales se centren en los cruces, los entramados y las continuidades de estas relaciones para seguir pensando la estructuración social.

La comida, atravesando nuestras vidas desde el día cero, se convierte en una realidad práctica a la vez que, como categoría cultural, se le cuelan todas las condiciones sociales que la sitúan geográfica, temporal y culturalmente. Por lo tanto, se vuelve imprescindible pensar la alimentación como el complejo sistema de relaciones socioculturales que es. Y pensar los cambios que en ella se producen, es pensar los cambios en los procesos de dominación, en la cultura, en la disponibilidad de los cuerpos y en la complejidad de las relaciones sociales que hacen posible cada plato de comida.

Referencias bibliográficas

- AGUIRRE, P. (2017) Una historia social de la comida. Buenos Aires: Lugar Editorial (2015). "La situación mundial». En: Aguirre, P., Díaz Córdova, D yPolischer, G. Cocinar y Comer en Argentina Hoy. Buenos Aires: Sociedad Argentina de Pediatría. (2010) La construcción social del gusto en el comensal moderno. En Katz, M., Aguirre, P. y Bruera, M. (2010) Comer. Puentes entre la alimentación y la cultura. Buenos Aires: Libros del Zorzal. ISBN: 978-987-599-154-5 (2009) Comida y comensales. Representaciones acerca de los alimentos, la comida y el cuerpo en diferentes sectores de ingreso del AMBA. En Buscando señales.Lecturas 
sobre nuevos hábitos del Consumo Cultural. Córdoba: Centro Cultural España-Córdoba.

(2006) Los alimentos rendidores y el cuerpo de

los pobres. En Millán Fuertes, A. A. (comp.) Arbitrario cultural: racionalidad e irracionalidad del comportamiento comensal. Homenaje a Igor de Garine. Barcelona: La Val de Onsera.

(2005) Estrategias de consumo: qué comen los argentinos que comen. Argentina: Miño y Dávila.

(2004) Ricos flacos y gordos pobres. La alimen-

tación en crisis. Buenos Aires: Capital Intelectual.

(1997). Patrón alimentario, estrategias de consu-

mo e identidad en la Argentina, 1995, en Procesos socioculturales y alimentación. Serie Antropológica. Ed. Del Sol, Buenos Aires, pp. 161-187.

- AGUIRRE, P.; DÍAZ CÓRDOVA, D. y POLISCHER, G. (2015) Cocinar y comer en Argentina hoy Buenos Aires: Fundasap y Sociedad Argentina de Pediatría. ISBN: 978-987-1279-23-4.

- Autor/a (2015) «Comer en la oficina: emociones, comensalidades y prácticas en torno "al comer» de trabajadores en el ámbito de la administración pública nacional", en Sánchez Aguirre, R. (Comp.) Sentidos y sensibilidades: exploraciones sociológicas sobre cuerpos-emociones. Ciudad Autónoma de Buenos Aires: Estudios Sociológicos Editora, pp. 183-202

- Autor/a y SORDINI, V. (2019) "Gustos y prácticas alimentarias de mujeres empleadas de oficinas públicas y mujeres destinatarias de programas alimentarios en Argentina", en Aposta. Revista de Ciencias Sociales. N`81, abril, mayo y junio 2019. ISSN 16967348. pp. 69-86.

- BOURDIEU, P. (2012 [1984]) La distinción. Criterio y bases sociales del gusto. Buenos Aires: Taurus.

- _ (2010) El sentido social del gusto. Elementos para una sociología de la cultura. Buenos Aires: Siglo Veintiuno Editores. (1998) La distinción. Criterios y bases sociales del

gusto. Madrid: Taurus

- BRUERA, M. (2010) Diet-éticas modernas. Razón, experiencia y resistencia alimentaria. En Katz, M., Aguirre, P. y Bruera, M. 
(2010) Comer. Puentes entre la alimentación y la cultura. Buenos Aires: Libros del Zorzal. ISBN: 978-987-599-154-5

(2006) La Argentina fermentada. Vino, alimenta-

ción y cultura. Buenos Aires: Paidós.

- CABRAL, X.; HUERGO, J. e IBAÑEZ, I. (2012) Políticas alimentarias en el avance de la frontera sojera: cuerpo(s) y disponibilidad(es) de la geometría colonial, en Papeles del CEIC, $n^{\circ} 78$, CEIC (Centro de Estudios sobre la Identidad Colectiva). Universidad del País Vasco. Disponible en: http://www.identidadcolectiva.es/pdf/78.pdf (en línea, nov.2017)

- CÁCERES NEVOT, J. y ESPEITX BERNAT, N. (2010) Comensales, consumidores y ciudadanos Una perspectiva sobre los múltiples significados de la alimentación en el siglo XXI.España: Montesinos.

- CERVIO, A. (2015) Experiencias en la ciudad y políticas de los sentidos. Lecturas sobre la vista, el oido y el olfato. En Sanchez Aguirre, R. (comp.) Sentidos y sensibilidades: exploraciones sociológicas sobre cuerpos/emociones. Buenos Aires: Estudios Sociológicos Editora.

- CONTRERAS HERNÁNDEZ, J. (2005) La modernidad alimentaria. Entre la sobreabundancia y la inseguridad, en Revista Internacional de Sociología (RIS), Tercera Época, $N^{\circ} 40$, enero-abril, 2005, pp. 109-132.

(1995) Alimentación y cultura. Necesidades, gustos

y costumbres. Barcelona: EdicionsUniversitat.

- CONTRERAS HERNÁNDEZ, J. y GRACIA-ARNÁIZ, M. (2005) Alimentación y cultura: perspectivas antropológicas. Barcelona: Ariel. - DE CASTRO, J. (1962) Geopolítica del hambre. Ensayo sobre los problemas alimentarios y demográficos del mundo. Buenos Aires: Solar Hachette.

- DE SENA, A. (2020) «Pobreza y programas sociales en la Argentina de las últimas décadas", en De Sena (comp) Vulnerabilidad, pobreza y políticas sociales: abanico de sentidos América Latina, Europa y China. (En prensa)

(2011). Promoción de microemprendimientos y políticas sociales: ¿Universalidad, focalización o masividad?, una 
discusión no acabada. Revista Pensamiento Plural, 8(1), 5-36. doi: 10.15210/pp.v0i8.68

- DÍAZ MÉNDEZ, C. (2005) Los debates actuales en la sociología de la alimentación. En Revista Internacional de Sociologia RIS. Tércera Época, N40, enero-abril 2005, pp. 47-78.

- DÍAZ-MÉNDEZ, C. Y GONZÁLEZ-ÁLVAREZ, M. (2013) La problematización de la alimentación: un recorrido sociológico por la publicidad alimentaria (1960-2010), en Empiria. Revista de Metodología de las Ciencias Sociales. N²5. enero-junio, 2013. pp. 121-145. Madrid, España.

- DOMANESCHI, L. (2012) Food social practices: Theory of practice and the new battlefieldof food quality, enJournal of Consumer Culture. SAGE publications.

- DURÁN-GUTIÉRRES, A.; Rodríguez-Weber, M.; De la TejaÁngeles, E. y Zebadúa-Penagos, M. (2012) Succión, deglución, masticación y sentido del gusto prenatales. Desarrollo sensorial temprano de la boca", en Acta PediatrícaMex, Vol. 33, №3, mayo-junio 2012. pp. 137-141. http://www.medigraphic.com/ pdfs/actpedmex/apm-2012/apm123g.pdf

- ELIAS, N. ([1939]2009) El proceso de la civilización. México: Fondo de Cultura Económica.

- EYNARD, M. (2017) Cuerpos y alimentación en crisis: conflictos sociales en torno a la cuestión alimentaria en la ciudad de Córdoba, 2001-2007. Buenos Aires: Ese Editora.

- FISCHLER, C. (2010) Gastro-nomía y gastro-anomía. Sabiduría del cuerpo y crisis biocultural de la alimentación moderna, en Gazeta de Antropología. N²6. ISSN 0214-7564-222

(1995) El (h)omnívoro. El gusto, la cocina y el cuerpo.

Barcelona: Anagrama

- GRASSI, E. (2003) «Políticas de Asistencia Focalizadas en el Desempleo y la Pobreza". En Políticas y problemas sociales en la sociedad neoliberal. La otra década infame (I). Buenos Aires: Espacio Editorial, pp. 221-302.

- GONZÁLEZ TURMO, I. (1997) Comida de rico, comida de pobre.

Evolución de los hábitos alimenticios en el occidente andaluz. Sevilla: Universidad de Sevilla. 
- GOODY, Jack (1995) Cocina, Cuisine y Clase: estudio de sociología comparada. México: Gedisa. ISBN 87-7432-549-8

- GOUDSBLOM, John (1995) Fuego y Civilización. Chile: Andrés Bello. - GRACIA-ARNAIZ, M. (2003) Alimentación y cultura: ¿hacia un nuevo orden alimentario? En Navas, J. y Gaona Pisoner, C. (coord.) Los modelos alimentarios a debate: la interdisciplinariedad de la alimentación. ISBN 84-932989-7-2, págs. 205-230. (1997) Aproximaciones para explicar el cambio alimentario. En Agricultura y sociedad. N82. Pp 153-182. España. ISSN 0211-8394

- HARRIS, M. (1985) Bueno para comer: enigmas de alimentación y cultura. Madrid: Alianza Editorial.

- HARVEY, D. (2013) Ciudades rebeldes. Del derecho de la ciudad a la revolución urbana. Madrid: Ediciones Akal.

- HUERGO, J. y BERTONE, J. (2014) Los circuitos y las circulaciones del comer y beber cordobés: entre los espectaculares viajes sensoriales a la "tierra prometida" y el rutinario caminar en círculos de encierro. En Boito, M. y Espoz, M. (Comp.) Urbanismo estratégico y separación clasista. Instantáneas de la ciudad en conflicto. Argentina: Puño y letra.

- JACINTO GARCÍA, L. (2013) Una historia comestible. Homínidos, cocina, cultura y ecología. Gijón: Trea.

- KATZ, Monica (2010) Comer: práctica individual, práctica social. En: Katz, M., Aguirre, P. y Bruera, M. (2010) Comer. Puentes entre la alimentación y la cultura. Buenos Aires: Libros del Zorzal. ISBN: 978-987-599-154-5.

- LAVA, M. (2012) Des-mitificando el dicho popular: "Sobre el gusto no hay nada escrito", En Cervio, A. (Comp) Las tramas del sentir. Ensayos desde una sociología de los cuerpos y las emociones. Buenos Aires: Estudios Sociológicos Editora, pp. 215-244. - LE BRETON, D. (2007) El sabor del mundo. Una antropología de los sentidos. Buenos Aires: Nueva Visión Argentina.

- LÉVI-STRAUSS, C. (1968). Mitológicas: lo crudo y lo cocido. México: Fondo de Cultura Económica.

- MONTANARI, M. (2004) La comida como cultura. Gijón: Ediciones TREA. 
- OMS/FAO (2003). Dieta, nutrición y prevención de enfermedades crónicas. Serie de Informes técnicos 916. Ginebra: OMS. - QUIJANO, Aníbal 2000 «Colonialidad del poder, eurocentrismo y América Latina" en Lander, Edgardo (comp.) La colonialidad del saber: eurocentrismo y ciencias sociales. Perspectivas latinoamericanas (Buenos Aires: CLACSO) p. 246.

- REMEDI, Fernando J., (2017). Modernidad alimentaria y afrancesamiento. Ciudad de Córdoba (Argentina) en el tránsito del siglo XIX al XX. En Historia Crítica, Nro. 65, pp. 71-92. doi: dx.doi. org/10.7440/histcrit65.2017.04 (2006). Dime qué comes y cómo lo comes y te diré quién eres. Una historia social del consumo alimentario en la modernización argentina. Córdoba, 1870-1930. Centro de Estudios Históricos "Prof. Carlos S. A. Segreti». Córdoba.

- RITZER, G. (1996) La McDonalización de la sociedad. Un análisis de la racionalización en la vida cotidiana. Barcelona: Ariel. - SCRIBANO, A. (2012) "Sociología de los cuerpos/emociones" en: Revista Latinoamericana de Estudios sobre Cuerpos, Emociones y Sociedad. RELACES. №10. Año 4. Diciembre 2012. marzo de 2013. Córdoba. ISSN: 1852.8759. pp. 93-113. Disponible en: http://www.relaces.com.ar/index.php/relaces/article/view/224 (2011) Un bosquejo conceptual del estado actual de la sujeción colonial. In: SINAIS - Revista Eletrônica - CiênciasSociais. Vitória: CCHN, UFES, Edição n.09, v.1, Junho. 2011. pp.43-75.

(2010) Primero hay que saber sufrir...!!! Hacia una sociología de la "espera" como mecanismo de soportabilidad social. En: Scribano, A.; Lisdero, P.

- (Eds.). Sensibilidades en juego: miradas múltiples desde los estudios sociales del cuerpo y las emociones. Córdoba: CEACONICET E-book, 2010. p. 169-194 (2007) Salud, dinero y amor! ... Narraciones de estudiantes universitarios sobre el cuerpo y la salud, en Onteaiken. Boletín sobre Prácticas y Estudios de Acción Colectiva, $\mathrm{N}^{\circ}$ 6. Programa de Estudios de Acción Colectiva y Conflicto Social. CIECSUE/ CONICET, Córdoba. Disponible 
en: http://www.accioncolectiva.com.ar/sitio/documentos/ ascribano2007a.pdf

- SORDINI (2018) “¡Nació con un PAN bajo el brazo! La transición a la democracia: entre el derecho y el subsidio a la alimentación", Unidad Sociológica I Número 12, Año 3 I febrero 2018-mayo 2018 I Buenos Aires. 